\title{
Supporting information for \\ Dynamical Correlation Effects on Photoisomerization: Ab Initio Multiple Spawning Dynamics with MS-CASPT2 for a Model trans-Pronated Schiff Base
}

\author{
Lihong Liu ${ }^{1,2,3}$, Jian Liu ${ }^{1,3,4}$ and Todd J. Martinez ${ }^{1,3, \dagger}$ \\ 1. Department of Chemistry and PULSE Institute, Stanford University, Stanford, California 94305, USA \\ 2. Key Laboratory of Theoretical and Computational Photochemistry, Ministry of Education, College of \\ Chemistry, Beijing Normal University, Beijing 100875, China \\ 3. SLAC National Accelerator Laboratory, Menlo Park, California 94309, USA. \\ 4. Beijing National Laboratory for Molecular Sciences, Institute of Theoretical and Computational Chemistry, \\ College of Chemistry and Molecular Engineering, Peking University, Beijing 100871, China
}

This document contains geometries in Cartesian coordinates and energies (in Angstrom and Hartree, respectively), optimized using state-averaged complete active space selfconsistent-field theory ${ }^{1-2}$ (SA-CASSCF) and multi-reference complete active space selfconsistent-field second order perturbation theory ${ }^{3}$ (MS-CASPT2). State averaging is performed over the lowest three singlet states with equal weighting, the active space contains six electrons in six orbitals, and the $6-31$ and $6-31 \mathrm{G}^{*}$ basis sets ${ }^{4-5}$ have been used. All of the calculations are performed using Molpro2006.2, ${ }^{6}$ including local modifications to calculate analytic nonadiabatic coupling vectors for MSPT2 wavefunctions. The package HDF_free (which is based on Molpro2006.2 and FMS90) is used for the dynamic simulation. Finally, we give a brief summary of seam space nudged elastic band method (SS-NEB). ${ }^{7}$

\section{Summary of Contents:}

Tables S1-S3

Tables S4-S6

Tables S7-S11

Tables S12-S22

Table S23

Table S24-S25

Figure S1

Figure S2

Section S1
Geometries of optimized $\mathrm{S}_{0}$ minima for trans-PSB3

Geometries of optimized $\mathrm{S}_{0}$ minima for $c i s$-PSB3

Geometries of optimized $\mathrm{S}_{1}$ minima for PSB3

Optimized MECI $\left(\mathrm{S}_{1} / \mathrm{S}_{0}\right)$ geometries for PSB3

Relative energies for PSB3 with CASSCF and MSPT2

Detailed Topography Parameters for CI Seams in Figs 4/5

$\mathrm{S}_{0} / \mathrm{S}_{1} / \mathrm{S}_{2}$ charge distributions for trans-PSB3 at $\mathrm{S}_{0}$ minimum

Twist angle and BLA trace along time at different levels

Brief summary of SS-NEB

$\uparrow$ Author to whom correspondence should be addressed. Electronic mail:

Todd.Martinez@stanford.edu 
Table S1. trans-PSB3 $\mathrm{S}_{0}$ state, SA3-CASSCF $(6,6) / 6-31 \mathrm{G}$ $\mathrm{E}\left(\mathrm{S}_{0}\right)=-248.16906212 ; \mathrm{E}\left(\mathrm{S}_{1}\right)=-247.98872740 ; \mathrm{E}\left(\mathrm{S}_{2}\right)=-247.96080158$

$\begin{array}{lrrr}\mathrm{C} & -6.0192702 & -0.7185904 & -0.0592481 \\ \mathrm{C} & -4.6640071 & -0.7396361 & 0.0078880 \\ \mathrm{H} & -6.5730076 & 0.2006016 & -0.0945302 \\ \mathrm{H} & -6.5892532 & -1.6249176 & -0.0800091 \\ \mathrm{H} & -4.1437760 & -1.6787369 & 0.0415145 \\ \mathrm{C} & -3.8766723 & 0.4637196 & 0.0366550 \\ \mathrm{C} & -2.5085577 & 0.4890888 & 0.1041349 \\ \mathrm{H} & -4.4128900 & 1.3972191 & 0.0022517 \\ \mathrm{H} & -1.9471799 & -0.4260184 & 0.1396261 \\ \mathrm{C} & -1.8152353 & 1.7206859 & 0.1280460 \\ \mathrm{H} & -2.3706433 & 2.6387237 & 0.0928148 \\ \mathrm{~N} & -0.5231805 & 1.8349417 & 0.1910250 \\ \mathrm{H} & -0.0734009 & 2.7271963 & 0.2057473 \\ \mathrm{H} & 0.0789281 & 1.0364048 & 0.2275579\end{array}$

Table S2. trans-PSB3 $\mathrm{S}_{0}$ state, SA3-MS-CASPT2(6,6)/6-31G $\mathrm{E}\left(\mathrm{S}_{0}\right)=-248.659212029 ; \mathrm{E}\left(\mathrm{S}_{1}\right)=-248.505173325 ; \mathrm{E}\left(\mathrm{S}_{2}\right)=-248.461213538$

$\begin{array}{lrrr}\mathrm{C} & -6.0483538 & -0.7287346 & -0.0607687 \\ \mathrm{C} & -4.6768375 & -0.7604095 & 0.0073764 \\ \mathrm{H} & -6.6013608 & 0.2110914 & -0.0959540 \\ \mathrm{H} & -6.6407138 & -1.6406081 & -0.0824934 \\ \mathrm{H} & -4.1501190 & -1.7161053 & 0.0413955 \\ \mathrm{C} & -3.8880122 & 0.4564575 & 0.0362771 \\ \mathrm{C} & -2.4969358 & 0.4847292 & 0.1048613 \\ \mathrm{H} & -4.4338968 & 1.4063407 & 0.0013577 \\ \mathrm{H} & -1.9244671 & -0.4455431 & 0.1410750 \\ \mathrm{C} & -1.8117133 & 1.7294880 & 0.1281360 \\ \mathrm{H} & -2.3758782 & 2.6632237 & 0.0922391 \\ \mathrm{~N} & -0.4852844 & 1.8541456 & 0.1926488 \\ \mathrm{H} & -0.0334313 & 2.7676254 & 0.2071861 \\ \mathrm{H} & 0.1288584 & 1.0389814 & 0.2301368\end{array}$


Table S3. trans-PSB3 $\mathrm{S}_{0}$ state, SA3-MS-CASPT2(6,6)/6-31G* $\mathrm{E}\left(\mathrm{S}_{0}\right)=-248.982323697 ; \mathrm{E}\left(\mathrm{S}_{1}\right)=-248.825737690 ; \mathrm{E}\left(\mathrm{S}_{2}\right)=-248.783168790$

$\begin{array}{lrrr}\mathrm{C} & -6.0198267 & -0.7048615 & -0.0595385 \\ \mathrm{C} & -4.6586598 & -0.7570852 & 0.0082853 \\ \mathrm{H} & -6.5525669 & 0.2418611 & -0.0938575 \\ \mathrm{H} & -6.6227442 & -1.6063074 & -0.0818053 \\ \mathrm{H} & -4.1420690 & -1.7137199 & 0.0418953 \\ \mathrm{C} & -3.8768935 & 0.4467655 & 0.0368417 \\ \mathrm{C} & -2.4949466 & 0.4714456 & 0.1050268 \\ \mathrm{H} & -4.4173308 & 1.3940486 & 0.0021107 \\ \mathrm{H} & -1.9191899 & -0.4518432 & 0.1412697 \\ \mathrm{C} & -1.8315843 & 1.7097193 & 0.1273575 \\ \mathrm{H} & -2.3980256 & 2.6381704 & 0.0914643 \\ \mathrm{~N} & -0.5218776 & 1.8477007 & 0.1909060 \\ \mathrm{H} & -0.0813215 & 2.7667388 & 0.2049976 \\ \mathrm{H} & 0.0988889 & 1.0380496 & 0.2285203\end{array}$

Table S4. cis-PSB3 $\mathrm{S}_{0}$ state, SA3-CASSCF(6,6)/6-31G

$\mathrm{E}\left(\mathrm{S}_{0}\right)=-248.16433461 ; \mathrm{E}\left(\mathrm{S}_{1}\right)=-247.98895661 ; \mathrm{E}\left(\mathrm{S}_{2}\right)=-247.95564471$

$\begin{array}{lrll}\mathrm{C} & -6.1682167 & 0.4316199 & 1.0013900 \\ \mathrm{C} & -4.8300110 & 0.4575223 & 1.2240710 \\ \mathrm{H} & -6.5770779 & 0.4147964 & 0.0091602 \\ \mathrm{H} & -6.8683912 & 0.4261405 & 1.8116627 \\ \mathrm{H} & -4.4771205 & 0.4731887 & 2.2377686 \\ \mathrm{C} & -3.8848267 & 0.4643892 & 0.1335775 \\ \mathrm{C} & -2.5132622 & 0.4897199 & 0.1899502 \\ \mathrm{H} & -4.3137719 & 0.4474700 & -0.8515460 \\ \mathrm{H} & -1.9600519 & 0.4911726 & -0.7303931 \\ \mathrm{C} & -1.7765244 & 0.5143264 & 1.3983976 \\ \mathrm{H} & -2.2794970 & 0.5139269 & 2.3443082 \\ \mathrm{~N} & -0.4792096 & 0.5384294 & 1.4475835 \\ \mathrm{H} & 0.0153155 & 0.5554829 & 2.3160784 \\ \mathrm{H} & 0.0827915 & 0.5410102 & 0.6190916\end{array}$


Table S5. cis-PSB3 $\mathrm{S}_{0}$ state, SA3-MS-CASPT2 $(6,6) / 6-31 \mathrm{G}$ $\mathrm{E}\left(\mathrm{S}_{0}\right)=-248.648214403 ; \mathrm{E}\left(\mathrm{S}_{1}\right)=-248.501656501 ; \mathrm{E}\left(\mathrm{S}_{2}\right)=-248.444383957$

$\begin{array}{lrrr}\mathrm{C} & -6.1892827 & 0.4309869 & 1.0044910 \\ \mathrm{C} & -4.8405782 & 0.4573800 & 1.2323606 \\ \mathrm{H} & -6.6006987 & 0.4141857 & -0.0050795 \\ \mathrm{H} & -6.9075135 & 0.4256286 & 1.8207048 \\ \mathrm{H} & -4.4766677 & 0.4734802 & 2.2609528 \\ \mathrm{C} & -3.8949384 & 0.4641574 & 0.1225517 \\ \mathrm{C} & -2.5078641 & 0.4897384 & 0.1751276 \\ \mathrm{H} & -4.3401542 & 0.4468485 & -0.8754075 \\ \mathrm{H} & -1.9430858 & 0.4912891 & -0.7591798 \\ \mathrm{C} & -1.7697133 & 0.5145330 & 1.4015067 \\ \mathrm{H} & -2.2827287 & 0.5141902 & 2.3630264 \\ \mathrm{~N} & -0.4492948 & 0.5389933 & 1.4558437 \\ \mathrm{H} & 0.0497498 & 0.5562861 & 2.3423824 \\ \mathrm{H} & 0.1229163 & 0.5414978 & 0.6118196\end{array}$

Table S6. cis-PSB3 $\mathrm{S}_{0}$ state, SA3-MS-CASPT2(6,6)/6-31G* $\mathrm{E}\left(\mathrm{S}_{0}\right)=-248.977519226 ; \mathrm{E}\left(\mathrm{S}_{1}\right)=-248.827447712 ; \mathrm{E}\left(\mathrm{S}_{2}\right)=-248.777577666$

$\begin{array}{lrrr}\mathrm{C} & -6.1650511 & 0.4313949 & 0.9983582 \\ \mathrm{C} & -4.8228633 & 0.4578858 & 1.2409080 \\ \mathrm{H} & -6.5585865 & 0.4146849 & -0.0142779 \\ \mathrm{H} & -6.8892437 & 0.4255951 & 1.8058958 \\ \mathrm{H} & -4.4679357 & 0.4738487 & 2.2684156 \\ \mathrm{C} & -3.8962101 & 0.4644028 & 0.1405366 \\ \mathrm{C} & -2.5086293 & 0.4898431 & 0.1838931 \\ \mathrm{H} & -4.3426798 & 0.4471353 & -0.8527732 \\ \mathrm{H} & -1.9497866 & 0.4912436 & -0.7499649 \\ \mathrm{C} & -1.7887310 & 0.5141097 & 1.3915500 \\ \mathrm{H} & -2.2903159 & 0.5140986 & 2.3550735 \\ \mathrm{~N} & -0.4724740 & 0.5384284 & 1.4474840 \\ \mathrm{H} & 0.0260136 & 0.5557491 & 2.3366903 \\ \mathrm{H} & 0.0966394 & 0.5407755 & 0.5993112\end{array}$


Table S7. PSB3 $\mathrm{S}_{1}$ minima, $\mathrm{S}_{1 \text { min_CenL }}$, SA3-MS-CASPT2 (6,6)/6-31G $\mathrm{E}\left(\mathrm{S}_{0}\right)=-248.584107398 ; \mathrm{E}\left(\mathrm{S}_{1}\right)=-248.519711531 ; \mathrm{E}\left(\mathrm{S}_{2}\right)=-248.407421010$

$\begin{array}{lrrr}\mathrm{C} & -5.7953118 & -0.8975788 & -0.6881912 \\ \mathrm{C} & -4.7775764 & -0.7324751 & 0.3478370 \\ \mathrm{H} & -5.5449356 & -0.7261650 & -1.7395249 \\ \mathrm{H} & -6.8401915 & -1.0999763 & -0.4332757 \\ \mathrm{H} & -4.6823802 & -1.4545927 & 1.1687308 \\ \mathrm{C} & -4.0012381 & 0.5522565 & 0.3301200 \\ \mathrm{C} & -2.5974924 & 0.5829203 & 0.1039052 \\ \mathrm{H} & -4.5786028 & 1.4804557 & 0.4176624 \\ \mathrm{H} & -2.0616836 & -0.3772406 & 0.0638187 \\ \mathrm{C} & -1.8368607 & 1.7750539 & -0.0808974 \\ \mathrm{H} & -2.3263582 & 2.7504886 & -0.0758246 \\ \mathrm{~N} & -0.4833142 & 1.7708711 & -0.2140599 \\ \mathrm{H} & 0.0343107 & 2.6411376 & -0.3007828 \\ \mathrm{H} & 0.0615652 & 0.9114271 & -0.2352388\end{array}$


Table S8. PSB3 $\mathrm{S}_{1}$ minima, $\mathrm{S}_{1 \text { min_CenL }}$, SA3-MS-CASPT2 $(6,6) / 6-31 \mathrm{G}^{*}$ $\mathrm{E}\left(\mathrm{S}_{0}\right)=-248.900991047 ; \mathrm{E}\left(\mathrm{S}_{1}\right)=-248.846742028 ; \mathrm{E}\left(\mathrm{S}_{2}\right)=-248.724991910$

$\begin{array}{lrrr}\mathrm{C} & -5.7082028 & -0.8791328 & -0.6929537 \\ \mathrm{C} & -4.7736516 & -0.7075832 & 0.3989937 \\ \mathrm{H} & -5.3876024 & -0.6914809 & -1.7177422 \\ \mathrm{H} & -6.7695850 & -1.0487327 & -0.5051512 \\ \mathrm{H} & -4.7029368 & -1.4411523 & 1.2080763 \\ \mathrm{C} & -4.0205794 & 0.5433499 & 0.3605115 \\ \mathrm{C} & -2.6245047 & 0.5848623 & 0.1099269 \\ \mathrm{H} & -4.5823206 & 1.4788433 & 0.4316992 \\ \mathrm{H} & -2.0980609 & -0.3728414 & 0.0370337 \\ \mathrm{C} & -1.8681103 & 1.7608428 & -0.0577283 \\ \mathrm{H} & -2.3388217 & 2.7415149 & -0.0114289 \\ \mathrm{~N} & -0.5377840 & 1.7378183 & -0.2516741 \\ \mathrm{H} & -0.0078350 & 2.6002723 & -0.3395668 \\ \mathrm{H} & -0.0100763 & 0.8700019 & -0.3057173\end{array}$

Table S9. PSB3 $\mathrm{S}_{1}$ minima, $\mathrm{S}_{1 \text { min_Cen }}$, SA3-CASSCF $(6,6) / 6-31 \mathrm{G}$ $\mathrm{E}\left(\mathrm{S}_{0}\right)=-248.07466091 ; \mathrm{E}\left(\mathrm{S}_{1}\right)=-248.05548533 ; \mathrm{E}\left(\mathrm{S}_{2}\right)=-247.94967633$

$\begin{array}{lrrr}\mathrm{C} & -5.9683172 & -0.8170926 & 0.1587440 \\ \mathrm{C} & -4.6206511 & -0.7989651 & 0.4175494 \\ \mathrm{H} & -6.5065419 & 0.0771922 & -0.0919678 \\ \mathrm{H} & -6.5335654 & -1.7266919 & 0.1993194 \\ \mathrm{H} & -4.0961545 & -1.6996155 & 0.6648920 \\ \mathrm{C} & -3.9175804 & 0.4117208 & 0.3737761 \\ \mathrm{C} & -2.4973737 & 0.5425187 & 0.6098950 \\ \mathrm{H} & -4.4900096 & 1.2891157 & 0.1071402 \\ \mathrm{H} & -1.8820182 & 0.4446500 & -0.2704262 \\ \mathrm{C} & -1.9511824 & 0.8043168 & 1.8219394 \\ \mathrm{H} & -2.5824684 & 0.9144472 & 2.6826157 \\ \mathrm{~N} & -0.6338300 & 0.9472079 & 2.0815276 \\ \mathrm{H} & -0.3146481 & 1.1397811 & 2.9980481 \\ \mathrm{H} & 0.0549975 & 0.8786039 & 1.3719293\end{array}$


Table S10. PSB3 $\mathrm{S}_{1}$ minima, $\mathrm{S}_{1 \mathrm{~min} \mathrm{CN}}, \mathrm{SA} 3-\mathrm{CASSCF}(6,6) / 6-31 \mathrm{G}$ $\mathrm{E}\left(\mathrm{S}_{0}\right)=-248.05702406 ; \mathrm{E}\left(\mathrm{S}_{1}\right)=-248.04530311 ; \mathrm{E}\left(\mathrm{S}_{2}\right)=-247.94422315$

$\begin{array}{lrrr}\mathrm{C} & -6.0428051 & -0.8524165 & -0.1053429 \\ \mathrm{C} & -4.6811225 & -0.7452428 & 0.0435135 \\ \mathrm{H} & -6.6596385 & 0.0071667 & -0.2855657 \\ \mathrm{H} & -6.5347886 & -1.8014804 & -0.0463563 \\ \mathrm{H} & -4.1125685 & -1.6414732 & 0.2217976 \\ \mathrm{C} & -3.9747240 & 0.4803695 & -0.0248182 \\ \mathrm{C} & -2.5672867 & 0.5621904 & 0.1321967 \\ \mathrm{H} & -4.5258332 & 1.3858961 & -0.2026619 \\ \mathrm{H} & -2.0289352 & -0.3526330 & 0.3100649 \\ \mathrm{C} & -1.8751319 & 1.7381431 & 0.0686079 \\ \mathrm{H} & -2.2925668 & 2.7116125 & -0.1019328 \\ \mathrm{~N} & -0.4477625 & 1.7561541 & 0.2361073 \\ \mathrm{H} & -0.0222180 & 1.8877572 & 1.1411691 \\ \mathrm{H} & 0.1789576 & 1.6777673 & -0.5504102\end{array}$

Table S11. PSB $3 \mathrm{~S}_{1}$ minima, $\mathrm{S}_{1 \text { min } \operatorname{Ter}}, \mathrm{SA} 3-\mathrm{CASSCF}(6,6) / 6-31 \mathrm{G}$ $\mathrm{E}\left(\mathrm{S}_{0}\right)=-248.06994659 ; \mathrm{E}\left(\mathrm{S}_{1}\right)=-24 \overline{8} .00329540 ; \mathrm{E}\left(\mathrm{S}_{2}\right)=-247.96251308$

$\begin{array}{lrrr}\mathrm{C} & -6.0783807 & -0.7508888 & -0.0669780 \\ \mathrm{C} & -4.6740550 & -0.6473391 & 0.0829874 \\ \mathrm{H} & -6.6208752 & -0.1818002 & -0.8065891 \\ \mathrm{H} & -6.6653202 & -1.4623695 & 0.4938144 \\ \mathrm{H} & -4.1816747 & -1.3505029 & -0.5926574 \\ \mathrm{C} & -3.9515680 & 0.1552977 & 0.8981738 \\ \mathrm{C} & -2.5167458 & 0.1757815 & 0.9674425 \\ \mathrm{H} & -4.4762055 & 0.8336654 & 1.5483216 \\ \mathrm{H} & -1.9618776 & -0.4887468 & 0.3320328 \\ \mathrm{C} & -1.8644863 & 1.0138306 & 1.8151650 \\ \mathrm{H} & -2.4294249 & 1.6740795 & 2.4460657 \\ \mathrm{~N} & -0.5243608 & 1.1149636 & 1.9606630 \\ \mathrm{H} & -0.1270610 & 1.7529233 & 2.6027997 \\ \mathrm{H} & 0.1034495 & 0.5586760 & 1.4344663\end{array}$

Table S12. PSB3 MECI $\left(\mathrm{S}_{1} / \mathrm{S}_{0}\right), \mathrm{MECI}_{\mathrm{Cen}}, \mathrm{SA3}-\mathrm{CASSCF}(6,6) / 6-31 \mathrm{G}$ $\mathrm{E}\left(\mathrm{S}_{0}\right)=-248.06368383 ; \mathrm{E}\left(\mathrm{S}_{1}\right)=-248.06368042 ; \mathrm{E}\left(\mathrm{S}_{2}\right)=-247.93594718$

$\begin{array}{lrrr}\mathrm{C} & -5.9308358 & -0.7534527 & -0.2256255 \\ \mathrm{C} & -4.5797885 & -0.5728905 & -0.2809921 \\ \mathrm{H} & -6.5475958 & -0.2579574 & 0.5002206 \\ \mathrm{H} & -6.4333116 & -1.4024588 & -0.9160321 \\ \mathrm{H} & -3.9754358 & -1.0719608 & -1.0081494 \\ \mathrm{C} & -3.9663501 & 0.3040226 & 0.6270435 \\ \mathrm{C} & -2.5045770 & 0.5601850 & 0.6806167 \\ \mathrm{H} & -4.6184607 & 0.7734804 & 1.3519217 \\ \mathrm{H} & -1.9974260 & -0.1255279 & 1.3372023 \\ \mathrm{C} & -1.8915222 & 1.5369478 & 0.0354533 \\ \mathrm{H} & -2.4491126 & 2.2080226 & -0.5915074 \\ \mathrm{~N} & -0.5534665 & 1.8191849 & 0.0613070 \\ \mathrm{H} & -0.1836469 & 2.5740732 & -0.4556856 \\ \mathrm{H} & 0.0754099 & 1.2877775 & 0.6088739\end{array}$

Table S13. PSB3 MECI $\left(\mathrm{S}_{1} / \mathrm{S}_{0}\right)$, MECI ${ }_{\mathrm{Cen}}$, SA3-MS-CASPT2 $(6,6) / 6-31 \mathrm{G}$ $\mathrm{E}\left(\mathrm{S}_{0}\right)=-248.568534227 ; \mathrm{E}\left(\mathrm{S}_{1}\right)=-248.568519529 ; \mathrm{E}\left(\mathrm{S}_{2}\right)=-248.443990302$

$\begin{array}{lrrr}\mathrm{C} & -6.2017318 & 1.3030090 & 0.7044865 \\ \mathrm{C} & -4.7990738 & 1.2677862 & 0.8065648 \\ \mathrm{H} & -6.7375750 & 0.6614653 & 0.0076054 \\ \mathrm{H} & -6.7918382 & 1.9778734 & 1.3159934 \\ \mathrm{H} & -4.3331735 & 1.9510542 & 1.5234105 \\ \mathrm{C} & -3.9601737 & 0.4199990 & 0.0594525 \\ \mathrm{C} & -2.4861023 & 0.4525999 & 0.1583692 \\ \mathrm{H} & -4.3652206 & -0.2453684 & -0.7105010 \\ \mathrm{H} & -1.8859341 & 1.1317047 & -0.4632746 \\ \mathrm{C} & -1.7601999 & -0.4123705 & 1.0527726 \\ \mathrm{H} & -2.2927394 & -1.1085124 & 1.6996609 \\ \mathrm{~N} & -0.4360803 & -0.3873279 & 1.1232681 \\ \mathrm{H} & 0.0812187 & -0.9967794 & 1.7602697 \\ \mathrm{H} & 0.1240926 & 0.2439689 & 0.5436891\end{array}$


Table S14. PSB3 MECI $\left(\mathrm{S}_{1} / \mathrm{S}_{0}\right)$, MECI $\mathrm{Cen}_{\text {, }}$ SA3-MS-CASPT2(6,6)/6-31G* $\mathrm{E}\left(\mathrm{S}_{0}\right)=-248.886166854 ; \mathrm{E}\left(\mathrm{S}_{1}\right)=-248.886162595 ; \mathrm{E}\left(\mathrm{S}_{2}\right)=-248.762916078$

$\begin{array}{lrrr}\mathrm{C} & -6.1740640 & 1.3194131 & 0.6940317 \\ \mathrm{C} & -4.7840222 & 1.2440841 & 0.8037816 \\ \mathrm{H} & -6.7185281 & 0.6974427 & -0.0072733 \\ \mathrm{H} & -6.7428956 & 2.0003056 & 1.3123605 \\ \mathrm{H} & -4.3097311 & 1.9095379 & 1.5265665 \\ \mathrm{C} & -3.9687421 & 0.3760170 & 0.0852248 \\ \mathrm{C} & -2.5106692 & 0.4338684 & 0.1700952 \\ \mathrm{H} & -4.3523158 & -0.2524634 & -0.7212988 \\ \mathrm{H} & -1.9038195 & 1.1155284 & -0.4365974 \\ \mathrm{C} & -1.7700471 & -0.4244107 & 1.0560275 \\ \mathrm{H} & -2.2801878 & -1.1381995 & 1.6967220 \\ \mathrm{~N} & -0.4679749 & -0.3597006 & 1.1172563 \\ \mathrm{H} & 0.0734225 & -0.9581200 & 1.7469314 \\ \mathrm{H} & 0.0650436 & 0.2957988 & 0.5379394\end{array}$

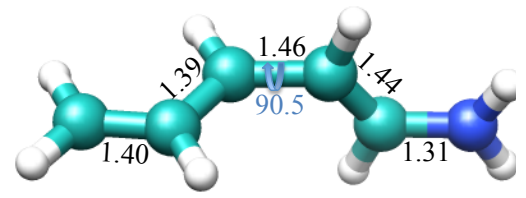

Table S15. PSB3 MECI $\left(\mathrm{S}_{1} / \mathrm{S}_{0}\right), \mathrm{MECI}_{\mathrm{BP}}, \mathrm{SA} 3-\mathrm{CASSCF}(6,6) / 6-31 \mathrm{G}$ $\mathrm{E}\left(\mathrm{S}_{0}\right)=-248.04499946 ; \mathrm{E}\left(\mathrm{S}_{1}\right)=-248.04496487 ; \mathrm{E}\left(\mathrm{S}_{2}\right)=-247.92928735$

$\begin{array}{lrrr}\mathrm{C} & -5.9374459 & -0.7865377 & -0.1627175 \\ \mathrm{C} & -4.6160739 & -0.5090833 & -0.3739797 \\ \mathrm{H} & -6.4879212 & -0.3560892 & 0.6525023 \\ \mathrm{H} & -6.4769047 & -1.4545324 & -0.8053310 \\ \mathrm{H} & -4.0780353 & -0.9308228 & -1.1961115 \\ \mathrm{C} & -3.9414856 & 0.2956889 & 0.5546342 \\ \mathrm{C} & -2.5260936 & 0.5452026 & 0.5316316 \\ \mathrm{H} & -4.5381705 & 0.6705212 & 1.3744069 \\ \mathrm{H} & -1.9956360 & -0.1691280 & 1.1464587 \\ \mathrm{C} & -1.8926004 & 1.5974295 & 0.0123731 \\ \mathrm{H} & -2.4506118 & 2.3375985 & -0.5355591 \\ \mathrm{~N} & -0.5591643 & 1.9076070 & 0.1818501 \\ \mathrm{H} & -0.0244224 & 2.1827073 & -0.5998392 \\ \mathrm{H} & -0.0515597 & 1.5412592 & 0.9442978\end{array}$


Table S16. PSB3 MECI $\left(\mathrm{S}_{1} / \mathrm{S}_{0}\right), \mathrm{MECI}_{\mathrm{CN}}, \mathrm{SA} 3-\mathrm{CASSCF}(6,6) / 6-31 \mathrm{G}$ $\mathrm{E}\left(\mathrm{S}_{0}\right)=-248.04293341 ; \mathrm{E}\left(\mathrm{S}_{1}\right)=-248.04292951 ; \mathrm{E}\left(\mathrm{S}_{2}\right)=-247.94135899$

$\begin{array}{lrrr}\mathrm{C} & -6.0501979 & -0.8957287 & -0.1034359 \\ \mathrm{C} & -4.6882344 & -0.7266700 & 0.0381160 \\ \mathrm{H} & -6.7042026 & -0.0642446 & -0.2835391 \\ \mathrm{H} & -6.4983765 & -1.8655578 & -0.0376984 \\ \mathrm{H} & -4.0904511 & -1.6054797 & 0.2173617 \\ \mathrm{C} & -4.0145920 & 0.5149350 & -0.0341563 \\ \mathrm{C} & -2.5964555 & 0.6176405 & 0.1223579 \\ \mathrm{H} & -4.5781142 & 1.4115687 & -0.2115743 \\ \mathrm{H} & -2.0627468 & -0.3026150 & 0.2991462 \\ \mathrm{C} & -1.9015115 & 1.7822897 & 0.0620924 \\ \mathrm{H} & -2.2180944 & 2.7901676 & -0.0995824 \\ \mathrm{~N} & -0.4164562 & 1.7266523 & 0.2458441 \\ \mathrm{H} & 0.0153379 & 1.8198051 & 1.1599323 \\ \mathrm{H} & 0.2176749 & 1.6110475 & -0.5384950\end{array}$



Table S17. PSB3 MECI $\left(\mathrm{S}_{1} / \mathrm{S}_{0}\right)$, MECI $\mathrm{MN}_{\mathrm{CN}}$, SA3-MS-CASPT2 $(6,6) / 6-31 \mathrm{G}$ $\mathrm{E}\left(\mathrm{S}_{0}\right)=-248.521729381 ; \mathrm{E}\left(\mathrm{S}_{1}\right)=-248.521723580 ; \mathrm{E}\left(\mathrm{S}_{2}\right)=-248.465958313$

$\begin{array}{lrrr}\mathrm{C} & -5.8565787 & -0.8909517 & -0.4530764 \\ \mathrm{C} & -4.6332557 & -0.7558471 & 0.1504180 \\ \mathrm{H} & -6.2978709 & -0.0905607 & -1.0480960 \\ \mathrm{H} & -6.4370024 & -1.8063664 & -0.3651591 \\ \mathrm{H} & -4.2251825 & -1.5715878 & 0.7513605 \\ \mathrm{C} & -3.8402252 & 0.4572561 & 0.0284562 \\ \mathrm{C} & -2.6457518 & 0.6476640 & 0.7002589 \\ \mathrm{H} & -4.2400328 & 1.2629935 & -0.5956185 \\ \mathrm{H} & -2.2226374 & -0.1596152 & 1.3103535 \\ \mathrm{C} & -1.8664716 & 1.9032362 & 0.6098424 \\ \mathrm{H} & -2.1548900 & 2.9027380 & 0.9322602 \\ \mathrm{~N} & -0.6687539 & 1.7274018 & -0.1902620 \\ \mathrm{H} & 0.1915819 & 1.3425438 & 0.2162527 \\ \mathrm{H} & -0.6893546 & 1.8449069 & -1.2106212\end{array}$


Table S18. PSB3 MECI $\left(\mathrm{S}_{1} / \mathrm{S}_{0}\right), \mathrm{MECI}_{\mathrm{CN}}$, SA3-MS-CASPT2 $(6,6) / 6-31 \mathrm{G}^{*}$ $\mathrm{E}\left(\mathrm{S}_{0}\right)=-248.842452213 ; \mathrm{E}\left(\mathrm{S}_{1}\right)=-248.842428925 ; \mathrm{E}\left(\mathrm{S}_{2}\right)=-248.781827882$

$\begin{array}{lrrr}\mathrm{C} & -5.7816282 & -0.8735991 & -0.4766806 \\ \mathrm{C} & -4.5745161 & -0.7319605 & 0.1444457 \\ \mathrm{H} & -6.2188437 & -0.0689377 & -1.0613607 \\ \mathrm{H} & -6.3459209 & -1.7975611 & -0.4105786 \\ \mathrm{H} & -4.1630135 & -1.5463251 & 0.7378622 \\ \mathrm{C} & -3.8267412 & 0.4858736 & 0.0476167 \\ \mathrm{C} & -2.6495669 & 0.6899018 & 0.7426217 \\ \mathrm{H} & -4.2302524 & 1.2905457 & -0.5673102 \\ \mathrm{H} & -2.2356256 & -0.1210764 & 1.3498401 \\ \mathrm{C} & -1.8728293 & 1.9208860 & 0.6329111 \\ \mathrm{H} & -2.1485941 & 2.9185481 & 0.9688253 \\ \mathrm{~N} & -0.7395164 & 1.7295289 & -0.1965309 \\ \mathrm{H} & 0.0485595 & 1.1688337 & 0.1406895 \\ \mathrm{H} & -0.8479367 & 1.7491535 & -1.2159821\end{array}$

Table S19. PSB3 MECI $\left(\mathrm{S}_{1} / \mathrm{S}_{0}\right), \mathrm{MECI}_{\mathrm{Ter}}, \mathrm{SA3}-\mathrm{CASSCF}(6,6) / 6-31 \mathrm{G}$ $\mathrm{E}\left(\mathrm{S}_{0}\right)=-247.99555591 ; \mathrm{E}\left(\mathrm{S}_{1}\right)=-247.99552900 ; \mathrm{E}\left(\mathrm{S}_{2}\right)=-247.885170030$

$\begin{array}{lrrr}\mathrm{C} & -6.0414254 & -0.8434804 & -0.1566651 \\ \mathrm{C} & -4.8223362 & -0.4366207 & 0.2857368 \\ \mathrm{H} & -6.4860946 & -0.4199958 & -1.0435055 \\ \mathrm{H} & -6.5299462 & -1.7089433 & 0.2626976 \\ \mathrm{H} & -4.5067949 & -1.2522495 & -0.5086122 \\ \mathrm{C} & -3.9812652 & 0.2855024 & 1.0264975 \\ \mathrm{C} & -2.5480765 & 0.2308412 & 1.0205360 \\ \mathrm{H} & -4.4583112 & 0.9807139 & 1.6965015 \\ \mathrm{H} & -2.0554596 & -0.4563124 & 0.3593642 \\ \mathrm{C} & -1.8197519 & 1.0359404 & 1.8388286 \\ \mathrm{H} & -2.3259337 & 1.7186086 & 2.4951015 \\ \mathrm{~N} & -0.4756059 & 1.0759145 & 1.9231188 \\ \mathrm{H} & -0.0209364 & 1.6937472 & 2.5475789 \\ \mathrm{H} & 0.1033516 & 0.4939042 & 1.3685292\end{array}$


Table S20. PSB3 MECI $\left(\mathrm{S}_{1} / \mathrm{S}_{0}\right)$, MECI $\mathrm{Mer}_{\text {er }}$ SA3-MS-CASPT2 $(6,6) / 6-31 \mathrm{G}$ $\mathrm{E}\left(\mathrm{S}_{0}\right)=-248.523119656 ; \mathrm{E}\left(\mathrm{S}_{1}\right)=-248.523113625 ; \mathrm{E}\left(\mathrm{S}_{2}\right)=-248.419930148$

$\begin{array}{lrrr}\mathrm{C} & -6.1062362 & -0.8369169 & -0.1573931 \\ \mathrm{C} & -4.8166891 & -0.4655673 & 0.2417741 \\ \mathrm{H} & -6.5781817 & -0.4022794 & -1.0491394 \\ \mathrm{H} & -6.6114819 & -1.7105494 & 0.2766190 \\ \mathrm{H} & -4.4604597 & -1.2772078 & -0.5394009 \\ \mathrm{C} & -3.9662131 & 0.2710590 & 1.0109074 \\ \mathrm{C} & -2.5325330 & 0.2215771 & 1.0105311 \\ \mathrm{H} & -4.4570673 & 0.9707561 & 1.6948927 \\ \mathrm{H} & -2.0168189 & -0.4709942 & 0.3407180 \\ \mathrm{C} & -1.8071572 & 1.0482788 & 1.8547568 \\ \mathrm{H} & -2.3308734 & 1.7387115 & 2.5189411 \\ \mathrm{~N} & -0.4465126 & 1.0916457 & 1.9463505 \\ \mathrm{H} & 0.0158872 & 1.7223658 & 2.5884879 \\ \mathrm{H} & 0.1457489 & 0.4966914 & 1.3776632\end{array}$

Table S21. PSB3 MECI $\left(\mathrm{S}_{1} / \mathrm{S}_{0}\right)$, MECI $\mathrm{Mer}_{\text {er }}$ SA3-MS-CASPT2 $(6,6) / 6-31 \mathrm{G}^{*}$ $\mathrm{E}\left(\mathrm{S}_{0}\right)=-248.851051190 ; \mathrm{E}\left(\mathrm{S}_{1}\right)=-248.850489075 ; \mathrm{E}\left(\mathrm{S}_{2}\right)=-248.747610470$

$\begin{array}{lrrr}\mathrm{C} & -6.0741616 & -0.8328939 & -0.1501115 \\ \mathrm{C} & -4.7921734 & -0.4203467 & 0.1947553 \\ \mathrm{H} & -6.5742026 & -0.4621276 & -1.0541529 \\ \mathrm{H} & -6.5450630 & -1.6934932 & 0.3467278 \\ \mathrm{H} & -4.4192834 & -1.2798499 & -0.4878973 \\ \mathrm{C} & -3.9589639 & 0.3026561 & 0.9834956 \\ \mathrm{C} & -2.5438128 & 0.2313091 & 0.9970494 \\ \mathrm{H} & -4.4489646 & 1.0174548 & 1.6420223 \\ \mathrm{H} & -2.0322336 & -0.4625198 & 0.3352372 \\ \mathrm{C} & -1.8257733 & 1.0412326 & 1.8495128 \\ \mathrm{H} & -2.3457200 & 1.7322508 & 2.5076967 \\ \mathrm{~N} & -0.4873621 & 1.0669793 & 1.9554709 \\ \mathrm{H} & -0.0244646 & 1.6842326 & 2.6096703 \\ \mathrm{H} & 0.1035927 & 0.4726861 & 1.3862312\end{array}$


Table S22. PSB3 MECI $\left(\mathrm{S}_{1} / \mathrm{S}_{0}\right)$, $\mathrm{MECI}_{\mathrm{CenR}}$, SA3-CASSCF(6,6)/6-31G $\mathrm{E}\left(\mathrm{S}_{0}\right)=-248.01073766 ; \mathrm{E}\left(\mathrm{S}_{1}\right)=-248.01072922 ; \mathrm{E}\left(\mathrm{S}_{2}\right)=-247.91423427$

$\begin{array}{lrrr}\mathrm{C} & -5.8660014 & -0.7202977 & 0.5423698 \\ \mathrm{C} & -4.6130607 & -0.6185937 & -0.0485497 \\ \mathrm{H} & -6.2618762 & 0.0664177 & 1.1546463 \\ \mathrm{H} & -6.4735318 & -1.5915063 & 0.4050046 \\ \mathrm{H} & -4.2566064 & -1.4306441 & -0.6550406 \\ \mathrm{C} & -3.8014966 & 0.5054177 & 0.1207133 \\ \mathrm{C} & -2.5485963 & 0.6082310 & -0.4972044 \\ \mathrm{H} & -4.1460547 & 1.3182091 & 0.7330672 \\ \mathrm{H} & -2.2186365 & -0.2275911 & -1.0936354 \\ \mathrm{C} & -1.6207198 & 1.7447037 & -0.3162129 \\ \mathrm{H} & -1.8621002 & 2.7065206 & -0.7263358 \\ \mathrm{~N} & -0.8116489 & 1.5892769 & 0.8309859 \\ \mathrm{H} & -0.8965375 & 2.2523503 & 1.5669505 \\ \mathrm{H} & 0.1093008 & 1.2336921 & 0.7042954\end{array}$

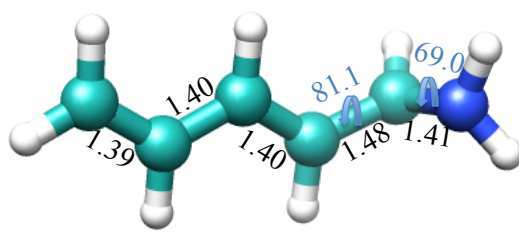


Table S23. Energies $(\mathrm{kcal} / \mathrm{mol})$ of equilibrium geometries of PSB3 using SA3CASSCF $(6,6) / 6-31 \mathrm{G}$ and SA3-MS-CASPT2 $(6,6) / 6-31 \mathrm{G}$ with varying numbers of uncorrelated (core) occupied orbitals. The zero of energy is defined by the $\mathrm{S}_{0}$ trans minimum.

\begin{tabular}{l|cccc}
\hline \hline $\begin{array}{c}\text { Core } \\
\text { Orbitals }\end{array}$ & MECI $_{\text {Cen }}$ & MECI $_{\text {Ter }}$ & MECI $_{\boldsymbol{C N}}$ & $\boldsymbol{S}_{\boldsymbol{0}^{-} \text {-trans }}$ \\
\hline $\mathbf{6}$ (default) & 56.9 & 85.4 & 86.3 & $0.0 / 96.7^{\mathrm{a}} / 124.2^{\mathrm{b}}$ \\
$\mathbf{1 3}$ & 57.3 & 91.4 & 95.2 & $0.0 / 101.2^{\mathrm{a}} / 127.2^{\mathrm{b}}$ \\
$\mathbf{1 4}$ & 55.7 & 93.3 & 93.7 & $0.0 / 102.1^{\mathrm{a}} / 127.9^{\mathrm{b}}$ \\
$\mathbf{1 5}$ & 57.8 & 92.1 & 93.1 & $0.0 / 103.7^{\mathrm{a}} / 128.6^{\mathrm{b}}$ \\
CASSCF & $\mathbf{6 6 . 1}$ & $\mathbf{1 0 8 . 9}$ & $\mathbf{7 9 . 1}$ & $0.0 / 113.2^{\mathrm{a}} / 130.7^{\mathrm{b}}$ \\
\hline \hline
\end{tabular}

${ }^{\mathrm{a}}$ Vertical excitation energy of $1^{\text {st }}$ excited state relative to $\boldsymbol{S}_{\mathbf{0}}$-trans.

${ }^{b}$ Vertical excitation energy of $2^{\text {nd }}$ excited state relative to $\boldsymbol{S}_{\boldsymbol{0}}$-trans. 
Table S24. Intersection topography characterization for CI seam beads connecting MECI $_{\mathbf{C e n}}$ and MECI $\mathbf{C N}_{\mathbf{C}}$ at the SA3-CASSCF $(6,6) / 6-31 \mathrm{G}$ level.

\begin{tabular}{|c|c|c|c|c|c|c|c|c|c|}
\hline Bead & $\begin{array}{c}\mathrm{C}=\mathrm{C}_{\mathrm{cen}} \\
\text { twist } \\
\text { (degrees) }\end{array}$ & $\begin{array}{c}\mathrm{C}=\mathrm{N} \\
\text { twist } \\
\text { (degrees) }\end{array}$ & $\begin{array}{c}\text { Energy } \\
(\mathrm{kcal} / \mathrm{mol})\end{array}$ & $\overline{s^{x}}$ & $\overline{s^{y}}$ & $\Delta_{g h}$ & $\overline{d_{g h}}$ & $s_{x}$ & $s_{y}$ \\
\hline 1 & 90.1 & 0.4 & 66.1 & -0.43 & -1.65 & 0.32 & 0.13 & -0.04 & -0.12 \\
\hline 2 & 90.2 & 4.1 & 66.2 & 0.70 & 1.48 & 0.45 & 0.13 & 0.08 & 0.10 \\
\hline 3 & 90.2 & 7.7 & 66.3 & 0.59 & 1.48 & 0.40 & 0.13 & 0.06 & 0.10 \\
\hline 4 & 90.1 & 10.9 & 66.5 & -0.01 & -1.56 & 0.29 & 0.13 & 0.00 & -0.12 \\
\hline 5 & 90.0 & 12.7 & 66.6 & -0.57 & 1.37 & 0.42 & 0.13 & -0.06 & 0.10 \\
\hline 6 & 89.9 & 15.1 & 66.9 & 0.71 & 0.72 & 0.75 & 0.15 & 0.10 & 0.04 \\
\hline 7 & 89.9 & 18.7 & 67.3 & -0.68 & -0.59 & 0.69 & 0.14 & -0.09 & -0.03 \\
\hline 8 & 90.1 & 22.5 & 68.0 & -0.51 & 0.22 & 0.80 & 0.16 & -0.08 & 0.01 \\
\hline 9 & 90.3 & 26.4 & 69.6 & -0.27 & 0.06 & 0.80 & 0.16 & -0.04 & 0.00 \\
\hline 10 & 90.9 & 29.2 & 77.1 & -1.07 & -0.07 & 0.66 & 0.11 & -0.11 & 0.00 \\
\hline 11 & 91.3 & 35.9 & 78.0 & -0.57 & -1.25 & 0.33 & 0.11 & -0.05 & -0.08 \\
\hline 12 & 91.2 & 37.5 & 79.1 & -0.01 & -1.82 & 0.44 & 0.10 & 0.00 & -0.10 \\
\hline 14 & 91.0 & 40.1 & 79.0 & -0.70 & 0.97 & 0.73 & 0.12 & -0.08 & 0.04 \\
\hline 15 & 91.1 & 41.8 & 80.0 & 0.77 & -0.15 & 0.69 & 0.11 & 0.08 & -0.01 \\
\hline 16 & 91.3 & 44.6 & 81.1 & 0.61 & -0.20 & 0.81 & 0.11 & 0.06 & -0.01 \\
\hline 17 & 91.6 & 47.6 & 83.0 & -0.38 & 0.58 & 0.68 & 0.11 & -0.04 & 0.03 \\
\hline 18 & 91.9 & 51.3 & 85.9 & -0.15 & -0.47 & 0.56 & 0.11 & -0.01 & -0.02 \\
\hline 19 & 92.2 & 55.7 & 90.5 & 0.27 & 0.24 & 0.41 & 0.10 & 0.02 & 0.01 \\
\hline 20 & 92.0 & 60.5 & 96.9 & -0.02 & -1.18 & 0.74 & 0.12 & 0.00 & -0.05 \\
\hline 21 & 90.8 & 65.2 & 104.8 & 0.09 & -2.11 & 0.75 & 0.12 & 0.01 & -0.09 \\
\hline 22 & 88.4 & 69.9 & 112.8 & 1.49 & 0.24 & 0.49 & 0.10 & 0.13 & 0.01 \\
\hline 23 & 85.2 & 74.3 & 119.5 & 0.47 & -3.21 & 0.59 & 0.10 & 0.04 & -0.15 \\
\hline 24 & 82.0 & 78.4 & 124.3 & 1.42 & -2.43 & 0.61 & 0.10 & 0.13 & -0.11 \\
\hline 25 & 78.9 & 82.2 & 127.9 & -1.54 & -2.70 & 0.44 & 0.09 & -0.12 & -0.13 \\
\hline 26 & 76.0 & 85.9 & 130.1 & 1.79 & -2.61 & 0.45 & 0.10 & 0.14 & -0.13 \\
\hline 27 & 72.7 & 88.6 & 131.3 & -2.04 & -2.55 & 0.07 & 0.09 & -0.14 & -0.16 \\
\hline 28 & 69.5 & 89.1 & 132.6 & -2.00 & -2.56 & -0.25 & 0.09 & -0.11 & -0.19 \\
\hline 29 & 66.3 & 89.2 & 133.9 & -1.61 & 2.96 & -0.16 & 0.09 & -0.10 & 0.21 \\
\hline 30 & 62.9 & 89.2 & 135.2 & 1.77 & -1.11 & 0.90 & 0.14 & 0.24 & -0.04 \\
\hline 31 & 59.4 & 89.3 & 136.6 & 2.60 & -2.22 & 0.52 & 0.10 & 0.23 & -0.11 \\
\hline 32 & 55.7 & 89.4 & 138.0 & -1.78 & -3.14 & -0.35 & 0.10 & -0.10 & -0.26 \\
\hline 33 & 51.7 & 89.6 & 139.4 & -2.06 & -1.82 & 0.89 & 0.14 & -0.29 & -0.06 \\
\hline 34 & 47.7 & 90.0 & 141.3 & -1.59 & 3.66 & -0.38 & 0.10 & -0.09 & 0.31 \\
\hline 39 & 36.4 & 91.4 & 83.8 & 0.57 & 0.90 & 0.36 & 0.10 & 0.05 & 0.05 \\
\hline 40 & 32.5 & 91.2 & 82.9 & 0.68 & -0.39 & 0.78 & 0.13 & 0.08 & -0.02 \\
\hline 41 & 28.2 & 91.1 & 82.1 & -0.71 & -0.60 & 0.77 & 0.13 & -0.08 & -0.03 \\
\hline 42 & 24.7 & 91.0 & 81.5 & 0.82 & 0.19 & 0.71 & 0.12 & 0.09 & 0.01 \\
\hline 43 & 21.5 & 90.9 & 81.0 & 0.68 & 1.26 & 0.46 & 0.11 & 0.06 & 0.07 \\
\hline 44 & 18.6 & 90.8 & 80.6 & 0.83 & 0.88 & 0.59 & 0.11 & 0.08 & 0.05 \\
\hline 45 & 15.8 & 90.7 & 80.2 & 0.74 & 1.21 & 0.52 & 0.11 & 0.07 & 0.06 \\
\hline 46 & 13.1 & 90.6 & 79.9 & 0.42 & 1.58 & 0.46 & 0.11 & 0.04 & 0.09 \\
\hline 47 & 10.4 & 90.5 & 79.6 & 0.27 & 1.67 & 0.46 & 0.11 & 0.03 & 0.09 \\
\hline 48 & 7.8 & 90.3 & 79.4 & 0.14 & 1.73 & 0.46 & 0.11 & 0.01 & 0.10 \\
\hline 49 & 5.3 & 90.2 & 79.3 & 0.10 & 1.75 & 0.47 & 0.11 & 0.01 & 0.10 \\
\hline 50 & 2.7 & 90.0 & 79.1 & -0.06 & -1.76 & 0.47 & 0.11 & -0.01 & -0.10 \\
\hline 51 & 0.0 & 90.0 & 79.1 & -0.85 & -0.64 & 0.68 & 0.12 & -0.09 & -0.03 \\
\hline
\end{tabular}


Table S25. Intersection topography characterization for CI seam beads connecting MECI $_{\text {Ter }}$ and MECI $\mathbf{I}_{\mathrm{Cen}}$ at the SA3-MS-CASPT2(6,6)/6-31G level with 14 core orbitals.

\begin{tabular}{|c|c|c|c|c|c|c|c|c|c|}
\hline Beads & $\begin{array}{c}\mathrm{C}=\mathrm{C}_{\mathrm{Ter}} \\
\text { twist } \\
\text { (degrees) }\end{array}$ & $\begin{array}{c}\mathrm{C}=\mathrm{C}_{\mathrm{Cen}} \\
\text { twist } \\
\text { (degrees) }\end{array}$ & $\begin{array}{c}\text { Energy } \\
(\mathrm{kcal} / \mathrm{mol})\end{array}$ & $\overline{s^{x}}$ & $\overline{s^{y}}$ & $\Delta_{g h}$ & $\overline{d_{g h}}$ & $s_{x}$ & $s_{y}$ \\
\hline 1 & 0.3 & 90.1 & 55.7 & 0.01 & 0.01 & 0.41 & 0.12 & 0.00 & 0.00 \\
\hline 2 & 2.6 & 89.3 & 56.6 & 0.04 & 0.04 & 0.54 & 0.13 & 0.00 & 0.00 \\
\hline 3 & 14.3 & 89.2 & 60.3 & 0.06 & 0.27 & 0.63 & 0.13 & 0.01 & 0.02 \\
\hline 4 & 33.1 & 88.7 & 71.2 & 0.22 & 0.39 & 0.45 & 0.12 & 0.02 & 0.02 \\
\hline 5 & 55.6 & 84.9 & 93.4 & -1.03 & 0.65 & 0.30 & 0.13 & -0.10 & 0.05 \\
\hline 6 & 77.6 & 70.2 & 110.0 & -0.95 & -1.26 & -0.86 & 0.12 & -0.03 & -0.14 \\
\hline 7 & 91.5 & 54.3 & 103.9 & 0.18 & -1.00 & 0.69 & 0.26 & 0.04 & -0.10 \\
\hline 8 & 91.0 & 33.9 & 99.0 & 0.18 & 0.97 & 0.59 & 0.21 & 0.03 & 0.09 \\
\hline 9 & 91.1 & 22.8 & 96.3 & -0.03 & 1.07 & 0.61 & 0.21 & -0.01 & 0.10 \\
\hline 10 & 90.8 & 11.9 & 94.5 & 0.52 & -0.25 & 0.59 & 0.21 & 0.10 & -0.02 \\
\hline 11 & 90.1 & 0.2 & 93.6 & -0.53 & -0.05 & 0.60 & 0.21 & -0.10 & 0.00 \\
\hline
\end{tabular}




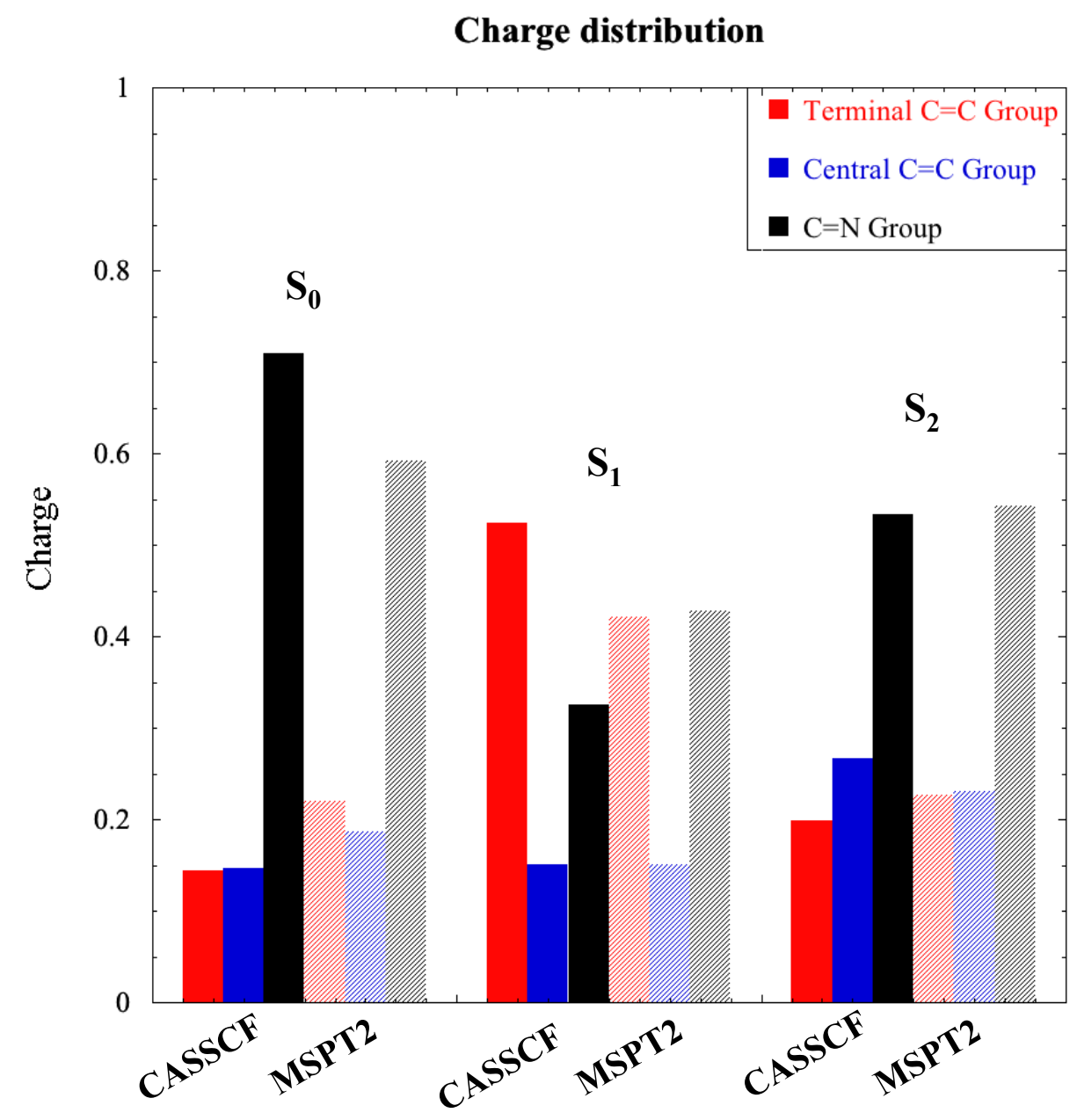

Figure S1. Charge distributions of trans-PSB3 $\left(\mathrm{S}_{0}\right.$ minimum) in different electronic states at the SA3-CAS $(6,6) / 6-31 \mathrm{G}$ and MS-SA3-CASPT2 $(6,6) / 6-31 \mathrm{G}$ levels. Charges on hydrogens are summed into heavy atoms. Terminal $\mathrm{C}=\mathrm{C}$ group consists of $\mathrm{C} 1$ and $\mathrm{C} 2$, central $\mathrm{C}=\mathrm{C}$ group consists of $\mathrm{C} 3$ and $\mathrm{C} 4$, and $\mathrm{C}=\mathrm{N}$ group consists of $\mathrm{C} 5$ and $\mathrm{N}$ (numbering follows Figure 1 in the main text). 
Figure S2. Time evolution of twist angles and bond length alternation from AIMSCASSCF and AIMS-MSPT2 dynamics. Averages are calculated over all TBFs which twist around the $\mathrm{C}=\mathrm{N}$ or terminal $\mathrm{C}=\mathrm{C}$ bond for AIMS-CASSCF and AIMS-MSPT2, respectively. Note that in both cases, these are the minor channels $(23 \%$ and $9 \%$ of the initial conditions for CASSCF/MSPT2, respectively). For both CASSCF and MSPT2 dynamics, the dominant outcome is torsion about the central $\mathrm{C}=\mathrm{C}$ bond.

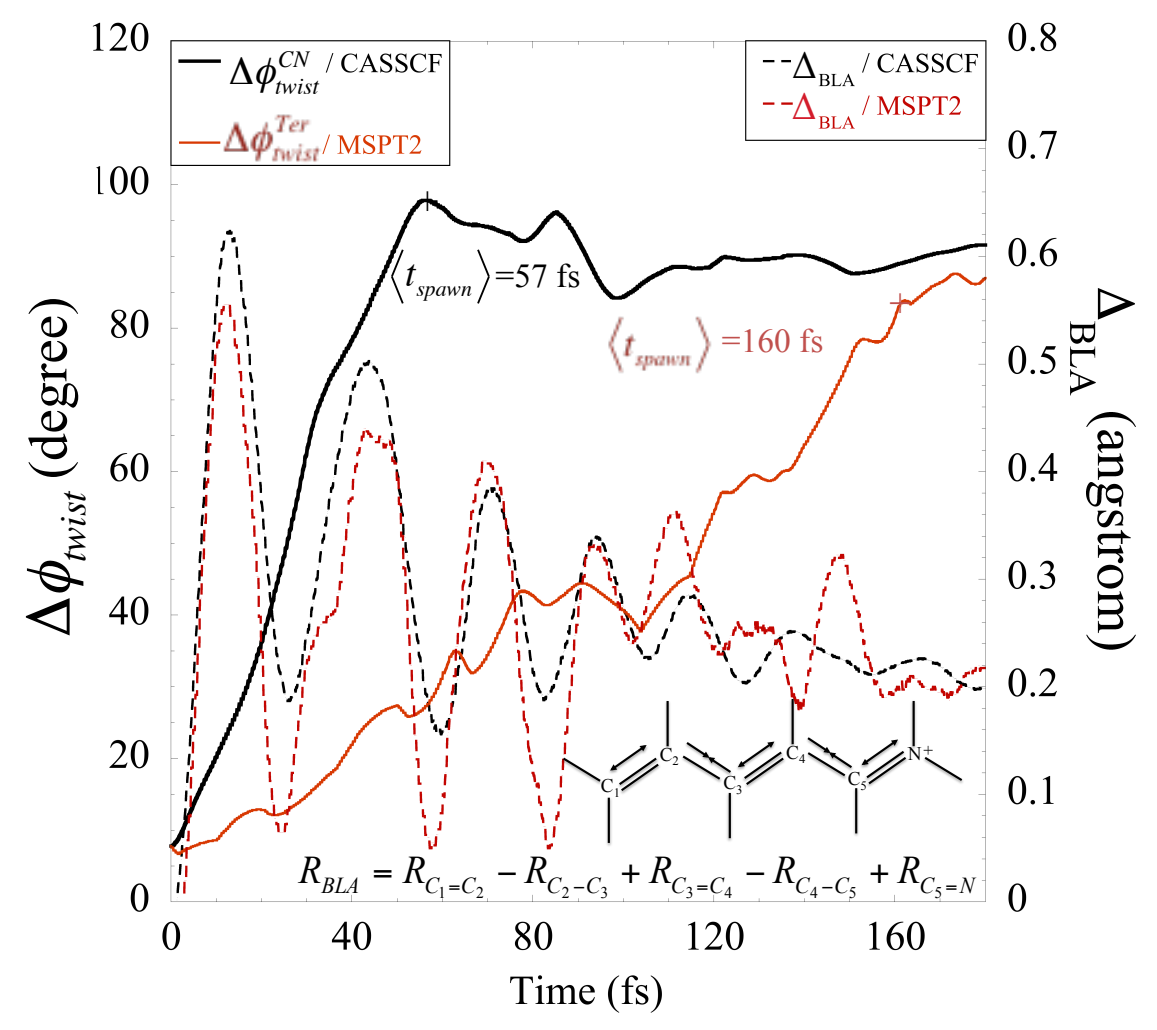


Section S1. Brief summary of seam space nudged elastic band (SS-NEB) method

We first define the energy difference gradient vector $\mathbf{g}$ and the non-adiabatic derivative coupling vector $\mathbf{h}$ as

$$
\begin{gathered}
\mathbf{g}_{I J}=\frac{\partial E_{I}}{\partial \mathbf{R}}-\frac{\partial E_{J}}{\partial \mathbf{R}} \\
\mathbf{h}_{I J}=\left(E_{J}-E_{I}\right)\left\langle\psi_{I}\left|\frac{\partial}{\partial \mathbf{R}}\right| \psi_{J}\right\rangle
\end{gathered}
$$

Here, I and J label the degenerate adiabatic electronic states. For the beads of the seam path, they should satisfy the two conditions

$$
\begin{aligned}
& \mathbf{g}_{I J} \cdot \delta \mathbf{R}=0 \\
& \mathbf{h}_{I J} \cdot \delta \mathbf{R}=0
\end{aligned}
$$

where $\delta \mathbf{R}$ is an infinitesimal geometry displacement away from the CI.

The total force that we minimize on the $i$ th bead with the SS-NEB method is

$$
\mathbf{f}_{i}\left(\mathbf{R}_{i}\right)=\mathbf{f}_{i, \| \tau}^{\text {spring }}\left(\mathbf{R}_{i}\right)+\mathbf{f}_{i, \perp g, h, \tau^{\prime}}\left(\mathbf{R}_{i}\right)+\mathbf{f}_{i, \| \mathbb{g}, h}^{\text {Egap }}\left(\mathbf{R}_{i}\right)
$$

Here $\tau^{\prime}$ is the tangent direction within the CI seam for the $i$ th molecular configuration (bead):

$$
\tau^{\prime}=\hat{\mathbf{P}}_{\perp g, h} \tau
$$

where $\tau$ represents the tangent direction of the path at the $i$ th point and $\hat{\mathbf{P}}_{\perp g, h}$ is the projection operator:

$$
\hat{\mathbf{P}}_{\perp g, h} \equiv \hat{I}-\mathbf{g g}^{T}-\mathbf{h h}^{T}
$$

$\mathbf{f}_{i, \| \tau}^{s p r i n g}$ is the harmonic spring force within the CI seam and $\mathbf{f}_{i, \perp g, h, \tau^{\prime}}$ is the component of the averaged potential energy surface gradient that is perpendicular to $\mathbf{g}, \mathbf{h}$, and $\tau$ ': 


$$
\begin{gathered}
\mathbf{f}_{i, \| \tau}^{\text {spring }}=\hat{\mathbf{P}}_{\perp g, h} \mathbf{F}_{i, \| \tau}^{\text {spring }} \\
\mathbf{f}_{i, \perp g, h, \tau^{\prime}}=\frac{1}{2} \nabla_{\perp g, h, \tau^{\prime}}\left(E_{J}+E_{I}\right)
\end{gathered}
$$

and

$$
\mathbf{f}_{i, \| \boldsymbol{g}, h}^{E g a p}=2\left(E_{J}-E_{I}\right) \mathbf{g}
$$




\section{Reference}

1. Werner, H.-J.; Knowles, P. J., A Second-Order Multiconfiguration SCF Procedure with Optimum Convergence. J. Chem. Phys. 1985, 82, 5053-5063.

2. Knowles, P. J.; Werner, H.-J., An Efficient Second-Order MCSCF Method for Long Configuration Expansions. Chem. Phys. Lett. 1985, 115, 259-267.

3. Finley, J.; Malmqvist, P.-A.; Roos, B. O.; Serrano-Andres, L., The Multi-State CASPT2 Method. Chem. Phys. Lett. 1998, 288, 299-306.

4. Hehre, W. J.; Ditchfield, R.; Pople, J. A., Self-Consistent Molecular Orbital Methods. Xii. Further Extensions of Gaussian-Type Basis Sets for Use in Molecular Orbital Studies of Organic Molecules. J. Chem. Phys. 1972, 56, 2257-2261. 5. Frisch, M. J.; Pople, J. A.; Binkley, J. S., Self-Consistent Molecular Orbital Methods. 25. Supplementary Functions for Gaussian Basis Sets. J. Chem. Phys. 1984, 80, 32653269.

6. Werner, H.-J.; Knowles, P. J.; Lindh, R.; Manby, F.; Schuetz, M. Molpro, V2006.2. 7. Mori, T.; Martinez, T. J., Exploring the Conical Intersection Seam: The Seam Space Nudged Elastic Band Method. J. Chem. Theo. Comp. 2013, 9, 1155-1163. 